\title{
The mass of the neutron star in Vela X-1 and tidally induced non-radial oscillations in GP Vel
}

\author{
H. Quaintrell ${ }^{1}$, A. J. Norton ${ }^{1}$, T. D. C. Ash ${ }^{1,2}$, P. Roche ${ }^{1,3,4}$, B. Willems ${ }^{1}$, T. R. Bedding ${ }^{5}$, \\ I. K. Baldry ${ }^{5,6}$, and R. P. Fender ${ }^{7}$
}

1 Department of Physics and Astronomy, The Open University, Walton Hall, Milton Keynes MK7 6AA, UK

2 Kildrummy Technologies Ltd, 1 Mill Lane, Lerwick, Shetland, ZE1 0AZ, UK

${ }^{3}$ Earth and Space Sciences, School of Applied Sciences, University of Glamorgan, Pontypridd CF37 1DL, UK

${ }^{4}$ Department of Physics and Astronomy, University of Wales, Cardiff CF24 3YB, UK

5 School of Physics, University of Sydney, New South Wales 2006, Australia

${ }^{6}$ Department of Physics and Astronomy, The Johns Hopkins University, 3400 North Charles Street, Baltimore, MD 21218-2686, USA

7 Sterrenkundig Instituut “Anton Pannekoek”, Kruislaan 403, 1098 SJ Amsterdam, The Netherlands

Received 30 September 2002 / Accepted 20 January 2003

\begin{abstract}
We report new radial velocity observations of GP Vel / HD 77581, the optical companion to the eclipsing X-ray pulsar Vela X-1. Using data spanning more than two complete orbits of the system, we detect evidence for tidally induced nonradial oscillations on the surface of GP Vel, apparent as peaks in the power spectrum of the residuals to the radial velocity curve fit. By removing the effect of these oscillations (to first order) and binning the radial velocities, we have determined the semiamplitude of the radial velocity curve of GP Vel to be $K_{\mathrm{o}}=22.6 \pm 1.5 \mathrm{~km} \mathrm{~s}^{-1}$. Given the accurately measured semi-amplitude of the pulsar's orbit, the mass ratio of the system is $0.081 \pm 0.005$. We are able to set upper and lower limits on the masses of the component stars as follows. Assuming GP Vel fills its Roche lobe then the inclination angle of the system, $i$, is $70.1^{\circ} \pm 2.6^{\circ}$. In this case we obtain the masses of the two stars as $M_{\mathrm{x}}=2.27 \pm 0.17 M_{\odot}$ for the neutron star and $M_{\mathrm{o}}=27.9 \pm 1.3 M_{\odot}$ for GP Vel. Conversely, assuming the inclination angle is $i=90^{\circ}$, the ratio of the radius of GP Vel to the radius of its Roche lobe is $\beta=0.89 \pm 0.03$ and the masses of the two stars are $M_{\mathrm{x}}=1.88 \pm 0.13 M_{\odot}$ and $M_{\mathrm{o}}=23.1 \pm 0.2 M_{\odot}$. A range of solutions between these two sets of limits is also possible, corresponding to other combinations of $i$ and $\beta$. In addition, we note that if the zero phase of the radial velocity curve is allowed as a free parameter, rather than constrained by the X-ray ephemeris, a significantly improved fit is obtained with an amplitude of $21.2 \pm 0.7 \mathrm{~km} \mathrm{~s}^{-1}$ and a phase shift of $0.033 \pm 0.007$ in true anomaly. The apparent shift in the zero phase of the radial velocity curve may indicate the presence of an additional radial velocity component at the orbital period. This may be another manifestation of the tidally induced non-radial oscillations and provides an additional source of uncertainty in the determination of the orbital radial velocity amplitude.
\end{abstract}

Key words. binaries: close - stars: neutron - stars: individual: Vela X-1 - stars: individual: GP Vel stars: individual: HD 77581 - stars: fundamental parameters

\section{Introduction}

Binary systems containing an X-ray pulsar are very important astrophysically as they can offer a direct measurement of the neutron star mass. The mass ratio of the system, $q$, is simply given by the ratio of the radial velocity semi-amplitudes for each component:

$q=\frac{M_{\mathrm{x}}}{M_{\mathrm{o}}}=\frac{K_{\mathrm{o}}}{K_{\mathrm{x}}}$

where $M_{\mathrm{x}}$ is the neutron star mass, $M_{\mathrm{o}}$ is the mass of the optical component, $K_{\mathrm{o}}$ is the semi-amplitude of the radial velocity of the optical component, and $K_{\mathrm{x}}$ is the semi-amplitude of the

Send offprint requests to: A. J. Norton,

e-mail: a.j.norton@open.ac.uk radial velocity of the neutron star. In addition, for an elliptical orbit, it can be shown that,

$M_{\mathrm{o}}=\frac{K_{\mathrm{x}}^{3} P\left(1-e^{2}\right)^{\frac{3}{2}}}{2 \pi G \sin ^{3} i}(1+q)^{2}$

and similarly,

$M_{\mathrm{x}}=\frac{K_{\mathrm{o}}^{3} P\left(1-e^{2}\right)^{\frac{3}{2}}}{2 \pi G \sin ^{3} i}\left(1+\frac{1}{q}\right)^{2}$

where $i$ is the inclination of the orbital plane to the line of sight, $e$ is the eccentricity and $P$ is the period of the orbit.

We therefore have a means of calculating the mass of the neutron star if the orbits of the two components and the inclination of the system are known. Such a combination is possible 
in an eclipsing X-ray binary system, in which the neutron star is a pulsar. X-ray pulse timing delays around the neutron star orbit yield the value of $K_{\mathrm{x}}$, and conventional radial velocity measurements from optical spectra yield $K_{\mathrm{o}}$.

A value for $i$ can be obtained from the following approximations:

$$
\begin{aligned}
& \sin i \approx \frac{\left(1-\beta^{2}\left(\frac{R_{\mathrm{L}}}{a^{\prime}}\right)^{2}\right)^{\frac{1}{2}}}{\cos \theta_{\mathrm{e}}} \\
& \frac{R_{\mathrm{L}}}{a^{\prime}} \approx A+B \log q+C \log ^{2} q
\end{aligned}
$$

where $R_{\mathrm{L}}$ is the radius of the optical companion's Roche lobe, $\beta=R_{\mathrm{o}} / R_{\mathrm{L}}$ is the ratio of the radius of the optical companion to that of its Roche lobe, $a^{\prime}$ is the separation between the centres of masses of the two components, and $\theta_{\mathrm{e}}$ is the eclipse halfangle. $A, B$, and $C$ have been determined by Rappaport \& Joss (1983) to be:

$$
\begin{aligned}
& A \approx 0.398-0.026 \Omega^{2}+0.004 \Omega^{3} \\
& B \approx-0.264+0.052 \Omega^{2}-0.015 \Omega^{3} \\
& C \approx-0.023-0.005 \Omega^{2}
\end{aligned}
$$

where $\Omega$ is the ratio of the rotational period of the companion star to the orbital period of the system. Note that, whilst $R_{\mathrm{L}} / a^{\prime}$ is expected to be constant for a given system, $R_{\mathrm{L}}, a^{\prime}$ and $\beta$ will vary around the orbit for a system which has an appreciable eccentricity.

Only seven eclipsing X-ray binary pulsars are known (namely Her X-1, Cen X-3, Vela X-1, SMC X-1, LMC X-4, QV Nor and OAO1657-415). Orbital parameters for the first six of these are still relatively poorly known, whilst the counterpart to OAO1657-415 has only recently been identified (Chakrabarty et al. 2002) and no optical radial velocity curve has been measured. In addition, the mass of the neutron star in a seventh eclipsing X-ray binary (4U1700-37) has recently been determined by Clark et al. (2002). This system does not contain an $\mathrm{X}$-ray pulsar though, and the mass determination is based on a Monte-Carlo modelling method which relies on the spectral type of the companion and is thus highly uncertain. Vela X-1 is the only one of these systems to have an eccentric orbit, and apart from OAO1657-415 is the one with the longest orbital period. For these reasons, and others discussed below, an accurate determination of the mass of the neutron star in Vela X-1 has always been difficult to obtain.

\section{The history of Vela $X-1$}

Vela X-1 was first detected by a rocket-borne experiment (Chodil et al. 1967) and subsequent observations (see, for example, Giacconi et al. 1972), suggested that the source was highly variable. Using data from the $O S O-7$ satellite, Ulmer et al. (1972) demonstrated evidence for intensity variations which were interpreted as eclipses with a period of around 9 days. An optical counterpart, GP Vel / HD 77581 (a B0.5 giant with $m_{v}=6.8$ ) was identified by Brucato \& Kristian (1972) and Hiltner et al. (1972), based on its ultra-violet excess and radial velocity variations.
Table 1. Measured amplitudes of the radial velocity curve of GP Vel.

\begin{tabular}{ll}
\hline \hline$K_{\mathrm{o}} / \mathrm{km} \mathrm{s}^{-1}$ & Reference \\
\hline $37-45$ & Wallerstein (1974) \\
$26 \pm 0.7$ & Zuiderwijk (1974) \\
$20 \pm 1$ & van Paradijs et al. (1976) \\
$21.75 \pm 1.15$ & van Paradijs et al. (1977) \\
$21.8 \pm 1.2$ & Rappaport \& Joss (1983) \\
$18.0-28.2$ & (95\% conf. range) van Kerkwijk et al. (1995) \\
$17.8 \pm 1.6$ & Stickland et al. (1997) \\
22 & (Correction to the above) Barziv et al. (2001) \\
$21.7 \pm 1.6$ & Barziv et al. (2001) \\
$22.6 \pm 1.5$ & this paper \\
\hline
\end{tabular}

An X-ray pulse period of $283 \mathrm{~s}$ was subsequently discovered using the SAS-3 satellite (Rappaport \& McClintock 1975; McClintock et al. 1976). Timing observations of these pulses by Rappaport et al. (1976) allowed the radial velocity semi-amplitude of the X-ray component to be measured as $K_{\mathrm{x}}=273 \pm 9 \mathrm{~km} \mathrm{~s}^{-1}$, and the eccentricity of the system, $e \sim 0.1$. Later work, using Hakucho and Tenma data (Deeter et al. 1987), gave a value of $a_{\mathrm{x}} \sin i=113.0 \pm 0.4$ light seconds for the projected semi-major axis. Using values for the orbital period $P$ and the eccentricity of the orbit $e$, the corresponding $K_{\mathrm{x}}$ value may be calculated according to

$K_{\mathrm{x}}=\frac{2 \pi}{P} \frac{a_{\mathrm{x}} \sin i}{\left(1-e^{2}\right)^{1 / 2}}$

as $K_{\mathrm{x}}=276 \pm 1 \mathrm{~km} \mathrm{~s}^{-1}$. The most recent, and accurate, values determined from $\mathrm{X}$-ray pulse timing analysis are those obtained with BATSE on board CGRO reported by Bildsten et al. (1997). They obtain a value $a_{\mathrm{x}} \sin i=113.89 \pm 0.13$ light seconds which corresponds to $K_{\mathrm{x}}=278.1 \pm 0.3 \mathrm{~km} \mathrm{~s}^{-1}$ when combined with their accurate values for the orbital period, $P=8.964368 \pm$ $0.000040 \mathrm{~d}$, and eccentricity, $e=0.0898 \pm 0.0012$.

The X-ray eclipse duration appears to be quite variable, and somewhat energy dependent. For example, Forman et al. (1973) obtained a value for the half angle of the eclipse of $\theta_{\mathrm{e}}=38^{\circ} \pm 1^{\circ}$ using the Uhuru satellite and Charles et al. (1976) obtained a value of $\theta_{\mathrm{e}}=39^{\circ} .8 \pm 00^{\circ} .4$ using the Copernicus satellite, whereas Watson \& Griffiths (1977) quote a value of $\theta_{\mathrm{e}}=33.8 \pm 1.3$ obtained using Ariel $V$ data. However the earlier two experiments were at much softer energies than Watson \& Griffiths observed, and softer X-rays are much more likely to be absorbed by circumstellar material, thus extending the observed eclipse time.

Early determinations of $K_{\mathrm{o}}$ were made by Wallerstein (1974) and Zuiderwijk et al. (1974), see Table 1. As regular $\mathrm{X}$-ray pulsations had yet to be discovered at this point, assumptions had to be made about the mass of the optical component in order to estimate the mass of the compact object. Zuiderwijk et al. (1974) obtained a value of $M_{\mathrm{x}}>2.5 \pm 0.3 M_{\odot}$, and suggested that such a large mass coupled with the lack of regular pulsations indicated that the compact object was a black hole. The discovery of regular X-ray pulsations provided a means of determining the masses of both components directly, and also ruled out the possibility of the compact object being a black hole. 
Van Paradijs et al. (1976) combined their $K_{\mathrm{o}}$ value obtained from 26 coudé spectrograms (Table 1) with the $K_{\mathrm{x}}$ value of Rappaport \& McClintock (1975). Using X-ray eclipse data, they determined that $i>74^{\circ}$, and thus arrived at a mass of $1.6 \pm 0.3 M_{\odot}$ for the neutron star. Van Paradijs et al. (1977) subsequently refined their $K_{\mathrm{o}}$ value using yet more photographic spectra (Table 1) and Rappaport \& Joss (1983) revised $K_{\mathrm{o}}$ further (Table 1) obtaining a neutron star mass estimate of $1.85_{-0.30}^{+0.35} M_{\odot}$ by combining data from a number of sources, including Watson \& Griffiths (1977), and Rappaport et al. (1980), and performing a Monte Carlo analysis to estimate the uncertainties.

More recently, van Kerkwijk et al. (1995) made further optical observations of GP Vel, and discovered strong deviations from a pure Keplerian velocity curve, which were autocorrelated within a single night, but not from one night to another. It was suggested that the variable gravitational force exerted by the neutron star as it travels around its eccentric orbit excites short-lived oscillations on the surface of the optical component which affect the measured radial velocity. From their $K_{\mathrm{o}}$ value (Table 1) van Kerkwijk et al. (1995) obtained $M_{\mathrm{x}}=1.9_{-0.5}^{+0.7} M_{\odot}$. A significantly lower value for $K_{\mathrm{o}}$ (Table 1 ) was obtained from observations using the IUE satellite by Stickland et al. (1997). However, Barziv et al. (2001) report that the analysis of these IUE data was subject to an error and a correct analysis yields a value consistent with those previously measured (Table 1) thus solving the discrepancy.

The most recent measurement of the optical radial velocity curve of GP Vel (Barziv et al. 2001) made use of 183 spectra obtained over a nine month campaign in order to try to average out the deviations reported by van Kerkwijk et al. (1995). Although they were quite successful in averaging out these excursions, they were left with different, phase-locked deviations in the radial velocity curve. Despite this they determined an accurate $K_{\mathrm{O}}$ value (Table 1) and set a limit on the neutron star mass of $M_{x} \sin ^{3} i=1.78 \pm 0.15 M_{\odot}$.

\section{Observations}

To take account of the deviations from Keplerian motion that are present in the radial velocity curve of GP Vel, we adopted a somewhat different approach to that employed by Barziv et al. (2001). Instead of trying to average the velocity excursions over many orbital periods, we chose to carry out a comprehensive radial velocity study with maximum phase coverage over several consecutive orbital cycles. In this way we aimed to track the velocity excursions closely and thus model and ultimately remove them.

The observations of GP Vel reported here were made using the 74-inch telescope at Mount Stromlo Observatory, Canberra, Australia. In order to cover two complete orbits, twenty-one continuous nights were obtained between 1996 February 1 and 1996 February 21. Only two nights were completely lost (the 9th and 17th) although most of the first night was spent trying different setup configurations, so this too produced little data. Typically, four sets of three spectra were obtained on each of the remaining nights, with exposures mostly of 1000s. In total 180 spectra of GP Vel were obtained.
The echelle and CCD camera were mounted at the coudé focus. All observations used the 31.6 lines $\mathrm{mm}^{-1}$ echelle grating, the 158 lines $\mathrm{mm}^{-1}$ cross disperser grating, the $81.3 \mathrm{~cm}$ focal length camera and the thinned $2 \mathrm{k} \times 2 \mathrm{k}$ Tektronix CCD, with a slit width of 2 arcsec. Around 70 separate echelle orders fell onto the detector spanning a wavelength range 3370$5970 \AA$. To shorten the readout time, $2 \times 2$ binning was used on the CCD for the first seven nights, but this was changed to $2 \times 1$ binning (i.e. binning only in the cross-dispersion direction) for the remainder of the run.

The radial velocity standards HR 1829 (a G5II star) and HR 3694 (a K5III-IV star) were also observed on most nights, as well as a B0Ia star ( $\epsilon$ Ori) to provide a template spectrum for the cross correlation. We also obtained the usual complement of flat fields and bias frames as well as thorium-argon arcs each time the telescope was moved.

\section{Data reduction and analysis}

The IRAF software package was used to perform the reduction and analysis. For each night's spectra, an average bias frame was constructed and subtracted from the arc, flat and object frames. Orders were traced automatically using the GP Vel frames, as the object was relatively bright and its earlytype spectrum does not contain many large absorption features which could complicate the tracing process. An optimal extraction algorithm was used to extract all the spectra in order to maximise the signal-to-noise ratio.

A wavelength calibration solution was found for the whole echelle frame using the arcs. A total of 1109 lines were identified from the thorium-argon arc spectrum (about 20 per order of the echelle) and the wavelength solution was fitted using a Chebyshev polynomial of order three in the wavelength direction plus another of order four in the spatial direction. The RMS of the fit was $0.005 \AA$, which is less than $10 \%$ of a pixel in all orders. The $F W H M$ of the individual arc lines near the centre of the echelle about $0.1 \AA$.

At the blue end, the signal-to-noise ratio was poor and there was also a problem with overlapping orders. Consequently only 53 orders were extracted from each echelle spectrum, spanning the wavelength range $3820 \AA$ to $5958 \AA$ (orders 148 to 96). The spectral resolution ranged from $0.06 \AA$ pixel $^{-1}$ at the blue end to $0.1 \AA$ pixel $^{-1}$ at the red end. All spectra were extracted onto a common log-linearised scale with a resolution of $4.86 \mathrm{~km} \mathrm{~s}^{-1}$ per pixel. An average spectrum is shown in Fig. 1.

The spectra of GP Vel showed essentially the same set of absorption lines as were seen by van Kerkwijk et al. (1995). In particular we saw the Balmer series from $\mathrm{H} \beta$ to $\mathrm{H} 9$, Her lines at $5015 \AA, 4921 \AA, 4713 \AA, 4471 \AA, 4387 \AA, 4143 \AA, 4121 \AA$ and $4026 \AA$, and several lines due to SiIII ( $4553 \AA$, $4568 \AA, 4575 \AA$,

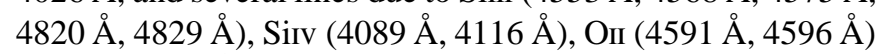
and NII (4601 $\AA$ ).

Before proceeding with the main cross-correlation, the stability of the detection system was checked by cross-correlating the various spectra of the radial velocity standards HR 1829 and HR 3694 against each other. Across the whole run, the mean shift in these spectra was only $0.46 \mathrm{~km} \mathrm{~s}^{-1}$, which is 

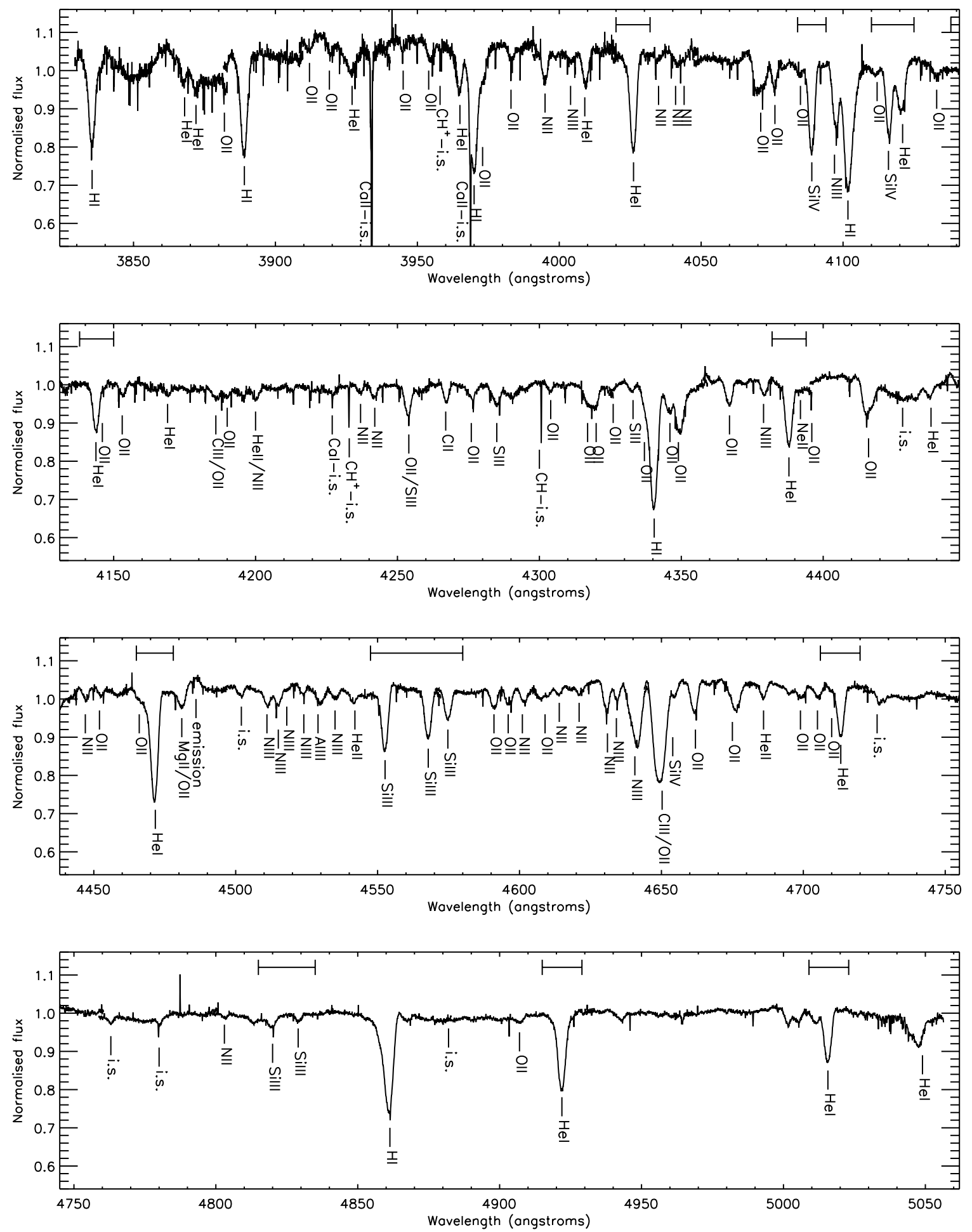

Fig. 1. An average spectrum of GP Vel. Horizontal bars indicate the regions used in the cross-correlation procedure. Many of the sharp lines peaking downwards are weak interstellar lines; most of the upward peaking lines are remaining sky features.

negligible $(<0.1$ of a pixel). As a further check, regions of the GP Vel spectra containing the CaII $3934 \AA$ and $\mathrm{NaI} 5890 / 5896 \AA$ interstellar lines were cross correlated against themselves. No trends were apparent in these data either.

To extract the radial velocity curve of GP Vel, template spectra of $\epsilon$ Ori were cross correlated against the spectra of the target. Since these stars have similar spectral types, systematic errors in this process should be minimised. Regions of the spectrum containing the Her lines plus the SirII and Sirv lines were used to produce the radial velocity curve in Fig. 2 (see Appendix for the individual radial velocity values, relative to $\epsilon$ Ori). The radial velocities themselves were extracted using standard IRAF routines by fitting a Gaussian to the cross-correlation function in each case. The Gaussian fits were 


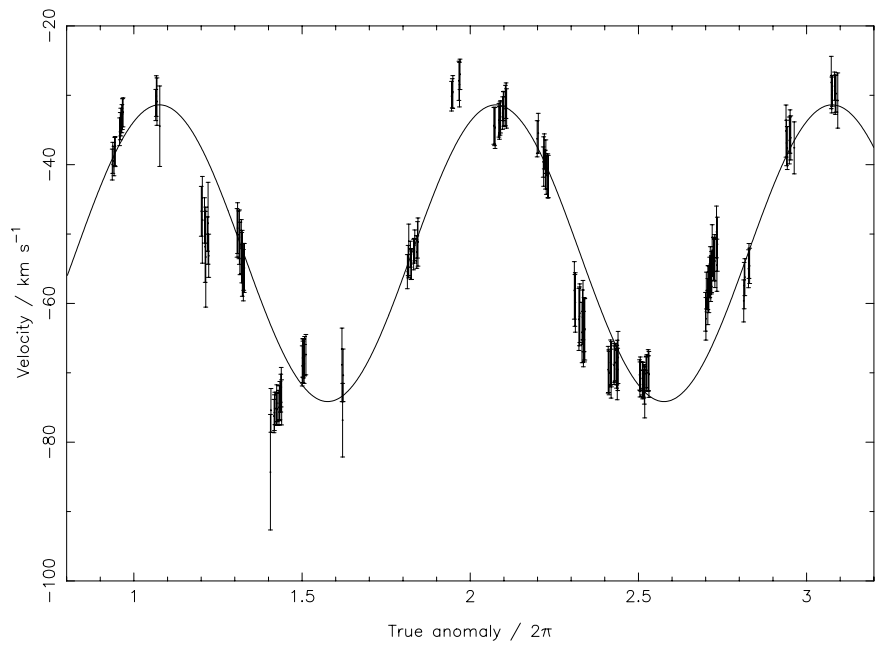

Fig. 2. Radial velocity curve for HeI, SiIII and Sirv lines showing the fitted curve (the first fit). Each cluster of radial velocity values represents a single night's data. The fit has a reduced chi-squared of 3.84 .

performed using the full shape of the Gaussian curve down to one-quarter of their height. Although radial velocities were also obtained from the Balmer lines, these lines were in general very broad and so it was difficult to obtain accurate velocities. Furthermore, there was some evidence that the radial velocity amplitude increased with the order of the Balmer line. We comment on this further below, but as a result did not use Balmer lines in the rest of our analysis.

\section{Fitting the radial velocity curve}

Because Vela X-1 is known to have a significant eccentricity, simply fitting a sinusoidal radial velocity curve to the crosscorrelation results as a function of time would not be satisfactory, as an eccentric orbit will show deviations from a pure sinusoid. Instead it may be shown that the observed radial velocity is given by:

$v=\gamma+\frac{4 \pi a_{1} \sin i}{P\left(1-e^{2}\right)^{\frac{1}{2}}} \frac{e \cos \omega+\cos (v+\omega)}{2}$

where $a_{1}$ is the semi-major axis of the orbit of GP Vel, $e$ is the orbital eccentricity, $P$ is the orbital period, $v$ is the true anomaly (i.e. the angle between the major axis and a line from the star to the focus of the ellipse), $\omega$ is the periastron angle (i.e. the angle between a line from the centre of the orbit to periastron and a line from the centre of the orbit to the ascending node), $i$ is the angle between the normal to the plane of the orbit and the line-of-sight and $\gamma$ is the radial velocity of the centre of mass of the binary system. The true anomaly is related to the eccentric anomaly, $E$, by:

$\tan \left(\frac{v}{2}\right)=\left(\frac{1+e}{1-e}\right)^{\frac{1}{2}} \tan \left(\frac{E}{2}\right)$

where $E$ is the angle between the major axis of the ellipse and the line joining the position of the object and the centre of the ellipse. In turn, $E$ can be related to $M$, the mean anomaly, by Kepler's Equation:

$E-e \sin E=M=\frac{2 \pi}{P}\left(t-T_{0}\right)$

where the right hand side of this equation is simply the orbital phase with $T_{0}$ the time of periastron passage. This equation can be solved numerically, or using Bessel functions.

The value of $T_{0}$ was obtained from the value of $T_{\pi / 2}$, the epoch of $90^{\circ}$ mean orbital longitude, derived by Bildsten et al. (1997) from BATSE pulse timing data. They give $T_{\pi / 2}=$ MJD 48 895.2186 \pm 0.0012 , from which

$T_{0}=T_{\pi / 2}+\frac{P\left(\omega-\frac{\pi}{2}\right)}{2 \pi}$

where $\omega=152.59^{\circ} \pm 0.92^{\circ}$. Hence we calculate the time of periastron passage as $T_{0}=$ MJD $48896.777 \pm 0.009$.

\subsection{The first fit}

Times, $t$, were assigned to each radial velocity measurement and Eq. (12) was solved using a numerical grid to calculate the corresponding eccentric anomaly. The true anomaly was then found using Eq. (11), and Fig. 2 shows a plot of radial velocity against true anomaly, with the best fit curve according to Eq. (10). The reduced chi-squared of the fit is $\chi_{\mathrm{r}}^{2}=3.84$. Scaling the error bars by a factor of 1.96 to reduce $\chi_{\mathrm{r}}^{2}$ to unity, gives the amplitude of the fitted curve as $K_{\mathrm{o}}=21.4 \pm 0.5 \mathrm{~km} \mathrm{~s}^{-1}$. However, we note that, since the use of chi-squared assumes that the errors on all the points are uncorrelated, the uncertainty here is likely to be a gross under-estimate. This will hereafter be referred to as the "first fit".

Figure 3 shows the residuals to the radial velocity curve in the first fit, plotted against time. It is clear that there are trends apparent in these residuals from night to night and a Fourier analysis shows that the dominant signals are at periods of $9 \pm 1 \mathrm{~d}$ and $2.18 \pm 0.04 \mathrm{~d}$ (Fig. 4). The $9 \mathrm{~d}$ signal present in the power spectrum reflects the fact that fixing the zero phase of the radial velocity curve, as implied by the X-ray data of Bildsten et al. (1997) does not provide the best fit to the data. We suggest this effect may be responsible for the "phase-locked" deviations revealed by Barziv et al. (2001) too. The $2.18 \mathrm{~d}$ modulation appears to be relatively stable throughout our two orbits of observations, with an amplitude of around $5 \mathrm{~km} \mathrm{~s}^{-1}$, as shown in Fig. 3.

\subsection{The second fit}

In order to take account of the correlated residuals from the first fit, another fit to the radial velocity data was performed this time with four free parameters: the $\gamma$ velocity and the $K_{\mathrm{o}}$ value as before, plus the amplitude and phase of a sinusoid with a period of $2.18 \mathrm{~d}$. The $K_{\mathrm{o}}$ part of the fit is sinusoidal as a function of true anomaly, whilst the $2.18 \mathrm{~d}$ part of the fit is sinusoidal purely as a function of time. The radial velocity fit according to this prescription, referred to as the second fit, is shown in Fig. 5 and has a reduced chi-squared of 2.40. The improvement over the first fit is therefore clearly apparent. Scaling the error bars 


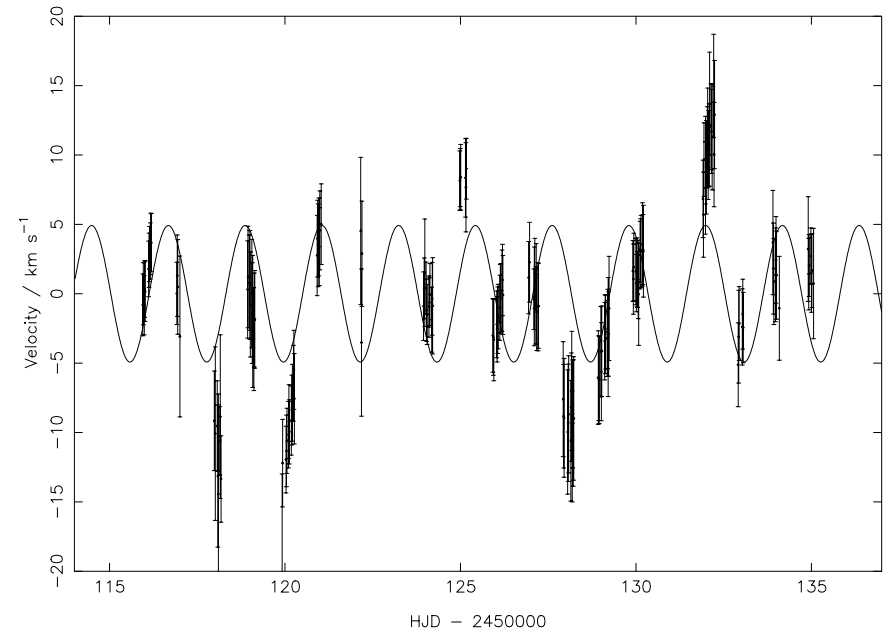

Fig. 3. Residuals to the radial velocity curve fit in Fig. 2, plotted against time. (The first fit.) Overlaid is a best-fit sinusoid with a period of $2.18 \mathrm{~d}$.

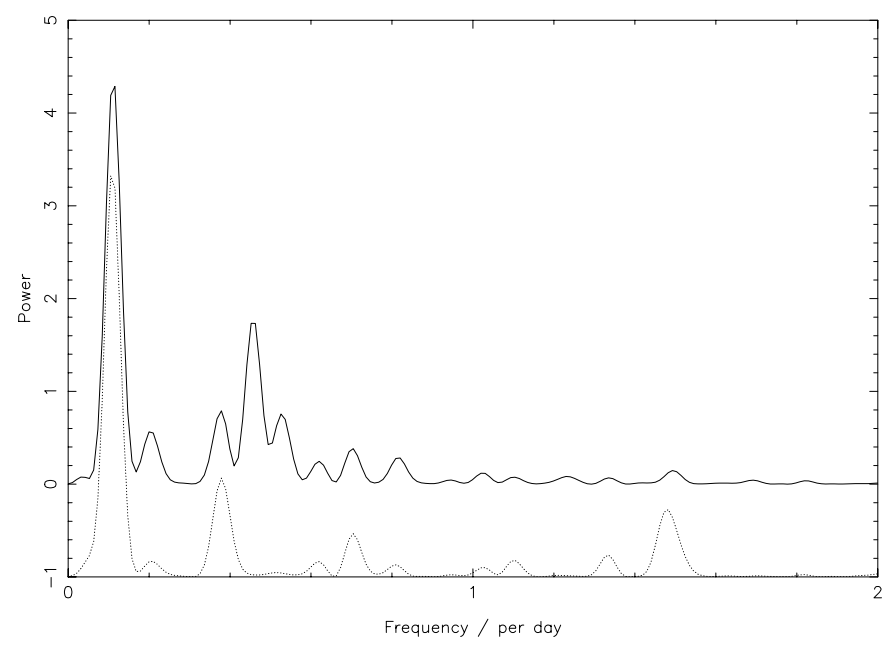

Fig. 4. Power spectra of the residuals to the radial velocity curve fits. The solid curve is the power spectrum of the data in Fig. 3 (the residuals after the first fit) and the dotted curve (offset in the -ve direction by one unit for clarity) is the power spectrum of the data in Fig. 6 (the residuals after the second fit). The highest peaks in the power spectrum of the residuals after the first fit correspond to frequencies of $0.111 \mathrm{~d}^{-1}$ and $0.459 \mathrm{~d}^{-1}$ or periods of $9.0 \mathrm{~d}$ and $2.18 \mathrm{~d}$. In each case, window function peaks due to the sampling of the lightcurve have been removed using a 1-dimensional clean (program courtesy H. Lehto).

by a factor of 1.55 to reduce $\chi_{\mathrm{r}}^{2}$ to unity, gives the amplitude of the fitted curve as $K_{\mathrm{o}}=22.4 \pm 0.5 \mathrm{~km} \mathrm{~s}^{-1}$ and the amplitude of the $2.18 \mathrm{~d}$ modulation as $5.4 \pm 0.5 \mathrm{~km} \mathrm{~s}^{-1}$. As with the first fit, we note that the uncertainties here are also likely to be under-estimates. The remaining residuals on the second fit are shown in Fig. 6, and their power spectrum is indicated by the dotted line in Fig. 4. It can be seen that some of the correlated structure in the residuals has been removed by this procedure, although the peak corresponding to a period of $\sim 9 \mathrm{~d}$ is still present indicating once again that the zero phase according to the X-ray observations does not provide the best description.

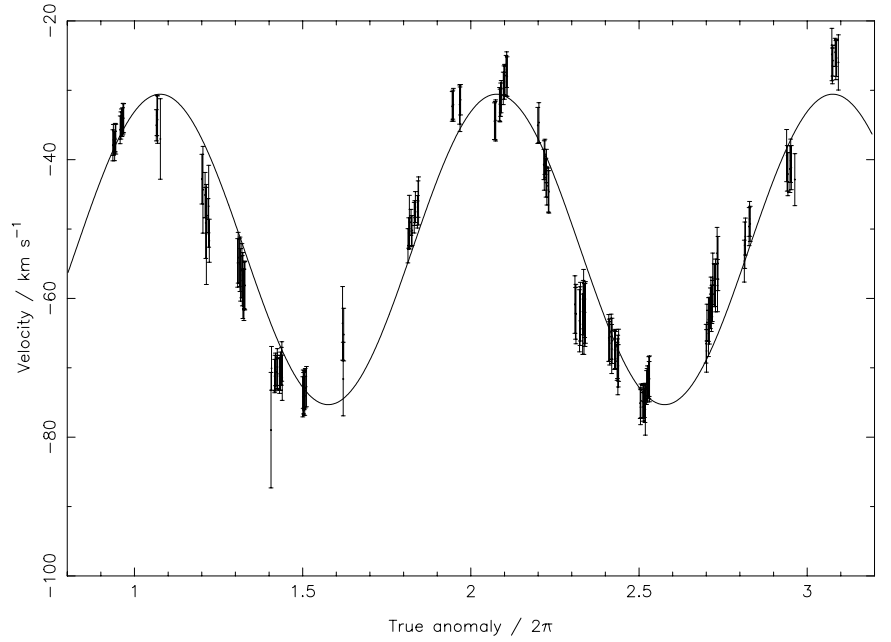

Fig. 5. Radial velocity curve for HeI, SirI and Sirv lines showing the fitted curve (the second fit) after removal of the 2.18 day signal. Each cluster of radial velocity values represents a single night's data. The fit has a reduced chi-squared of 2.40 .

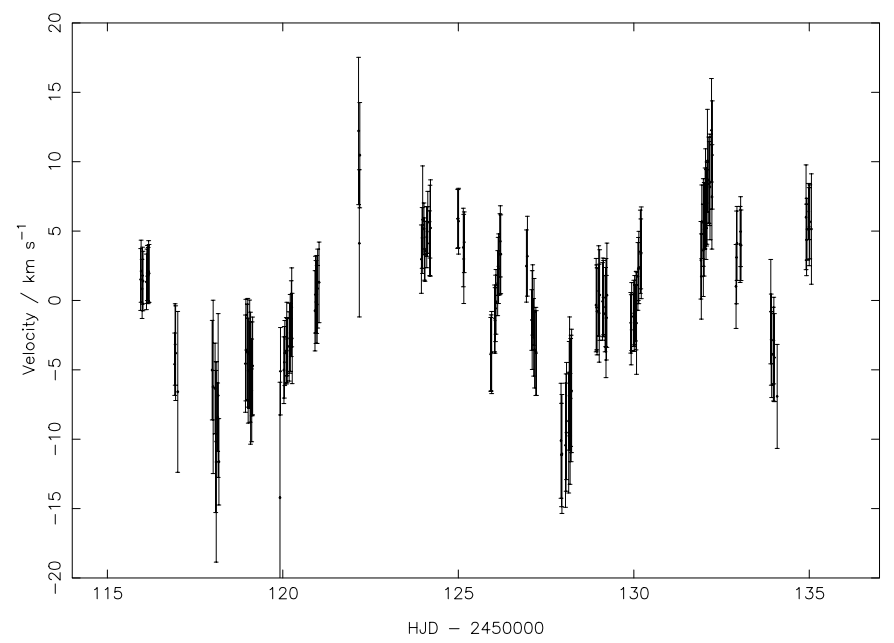

Fig. 6. Residuals to the radial velocity curve fit in Fig. 5, plotted against time. (The second fit.)

\subsection{Fits to phase binned means}

To test whether the deviations from the radial velocity curve displayed by each group of data points are correlated, we subtracted the effect of the $2.18 \mathrm{~d}$ period according to the parameters found for it in the second fit, and rebinned the resulting radial velocities into nine phase bins (each $\sim 1$ day). The radial velocity fit to these phase-binned means is shown in Fig. 7 and has a reduced chi-squared of 28.3. The errors on the mean data points are calculated as the standard error on the mean from the individual data points that are averaged in each case. The fact that the reduced chi-squared is significantly greater than unity suggests that the deviations are indeed correlated. Scaling the error bars here by a factor of 5.3 to reduce $\chi_{\mathrm{r}}^{2}$ to unity, gives the amplitude of the fitted curve as $K_{\mathrm{o}}=22.6 \pm 1.5 \mathrm{~km} \mathrm{~s}^{-1}$.

Figure 7 clearly shows that a better fit would be obtained if the zero phase of the radial velocity is allowed as a free parameter. This reflects the fact that $\mathrm{a} \sim 9 \mathrm{~d}$ period is present in 


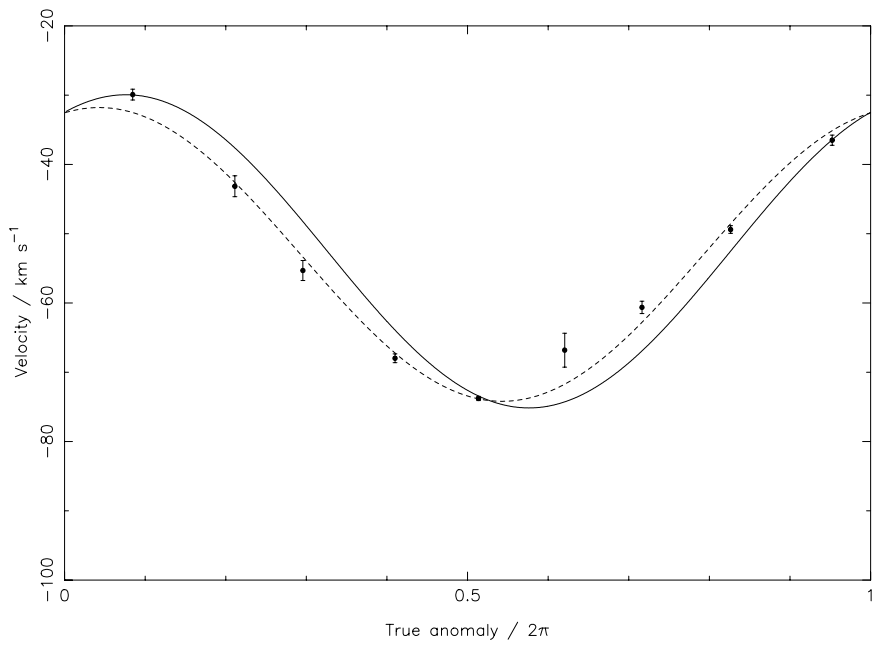

Fig. 7. Radial velocity curve for HeI, SirII and Sirv lines binned into nine phase bins. Each radial velocity value represents two or more night's data. The fit to the phase binned means has a reduced chisquared of 28.3 and is shown by the solid curve. A second fit to the phase binned means, allowing the zero phase of the radial velocity as a free parameter, is shown by the dashed line and has a reduced chi-squared of 6.0 .

the power spectra of the residuals after both the first and second fits (Fig. 4). Allowing the zero phase to vary results in the second fit to the phase binned data shown by the dashed line in Fig. 7. This time the reduced chi-squared is 6.0. Scaling the error bars here by a factor of 2.44 to reduce $\chi_{\mathrm{r}}^{2}$ to unity, gives the amplitude of the fitted curve as $21.2 \pm 0.7 \mathrm{~km} \mathrm{~s}^{-1}$. The shift of the minimum radial velocity with respect to that in the first fit to the pase binned means is a true anomaly of $\Delta v=0.033 \pm 0.007$, corresponding to about 7 hours. We note that this shift in zero phase is significantly greater than the uncertainty implied by extrapolating the X-ray ephemeris of Bildsten et al. (1997) to the epoch of our observations which is only $\sim 0.0006$ of a cycle. We conclude that the orbital phase locked deviations remaining in the radial velocities after the first fit to means can therefore mostly be accounted for by a simple phase shift in the radial velocity curve.

\section{Calculation of system parameters}

The masses of the two stellar components were calculated according to the method described in Sect. 1. In order to propagate the uncertainties, a Monte Carlo approach was adopted, calculating $10^{6}$ solutions with input values drawn from a Gaussian distribution within the respective $1 \sigma$ uncertainties of each parameter. The final values were then calculated as the mean result of these $10^{6}$ solutions, with the $1 \sigma$ uncertainties given by the rms deviation in each case.

The procedure was carried out using $K_{\mathrm{o}}=22.6 \pm 1.5 \mathrm{~km} \mathrm{~s}^{-1}$ from the first fit to means and the results of these calculations are shown in Table 2. We used the value of $K_{\mathrm{x}}=$ $278.1 \pm 0.3 \mathrm{~km} \mathrm{~s}^{-1}$ (Bildsten et al. 1997), to calculate $q$ using Eq. (1) and a value of $33^{\circ} .8 \pm 1.3^{\circ}$ was adopted for the eclipse half-angle, $\theta_{\mathrm{e}}$ from Watson $\&$ Griffiths (1977). We note that this value was also used by Wilson \& Terrel (1998) for their unified analysis of Vela X-1 and appears to be the most reliable. A value of $0.67 \pm 0.04$ was adopted for the co-rotation factor, $\Omega$, obtained from comparison of GP Vel spectra with model line profiles by Zuiderwijk (1995). We note that Barziv et al. (2001) also used the $v \sin i$ value derived by Zuiderwijk (1985) and obtained a similar value, namely $\Omega\left(=f_{\text {co }}\right)=0.69 \pm 0.08$.

Values for $\beta$ and $i$ cannot be obtained from independent observations. However, since the supergiant is unlikely to be overfilling its Roche lobe, an upper limit to the Roche lobe filling factor is $\beta \leq 1.0$; and an upper limit to the inclination is clearly $i \leq 90^{\circ}$. A lower limit to $\beta$ is found by setting $i=90^{\circ}$, and a lower limit to $i$ is found by setting $\beta=1$. These limits are indicated in Table 2 . We note though that $\beta$ is unlikely to have a fixed value, as the size and shape of the Roche lobes of both components will vary as the separation between the stars varies as they orbit each other eccentrically. Indeed, it has been suggested that GP Vel is likely to fill its Roche lobe at periastron (Zuiderwijk 1995). However the mass ratio implies that Roche lobe overflow would be dynamically unstable and lead to a common-envelope phase, so the limit of $\beta=1$ is probably implausible. We also note that Barziv et al. (2001) took a somewhat different approach and assumed a filling factor at periastron in the range $\beta=0.9-1.0$, thus leading to a minimum value for the inclination of $73^{\circ}$, consistent with our analysis.

Given these two limits on each of $\beta$ and $i$, Eqs. (2) and (3) then allow us to calculate the masses of the two components for combinations of $i$ and $\beta$ between the two extremes. The limiting values for the masses of the two stars are shown in Table 2. Values between these extremes, as a function of inclination angle, are shown in Fig. 8. With these two limiting situations, we also calculate the semi major axis $a$ of the orbit from Kepler's law, and this is then used to calculate the separation of the stars at periastron according to $a^{\prime}=a(1-e)$. The Roche lobe radius at periastron $R_{\mathrm{L}}$ is then determined from the value of $R_{\mathrm{L}} / a^{\prime}$ at each extreme, and the radius of the companion star $R_{\mathrm{O}}$ is then calculated as $\beta \times R_{\mathrm{L}}$ in each case. These values too are shown in Table 2 . For each parameter, the actual value may be anywhere within the range encompassed by the two limits.

\section{Tidally induced non-radial oscillations}

The strongest peaks in the power spectrum of the residuals after the first fit, at $0.111 \mathrm{~d}^{-1}$ and $0.457 \mathrm{~d}^{-1}$, are suggestively close to the orbital frequency and $4 \times$ the orbital frequency $\left(f_{\text {orb }}=\right.$ $0.11155 \mathrm{~d}^{-1}$ ) respectively. This leads us to speculate that they may both represent tidally excited non-radial oscillation modes (see also Willems \& Aerts 2002; Handler et al. 2002).

From a theoretical point of view, the tidal action exerted by one binary component on the other is governed by the tidegenerating potential which can be expanded in a Fourier series in terms of multiples of the mean motion $n=2 \pi f_{\text {orb }}$ (e.g. Polfliet \& Smeyers 1990). The oscillations that are most likely to be excited are those associated with the lower-order harmonics of the Fourier series. In the particular case of GP Vel, the dominant contributions to the Fourier series of the tidegenerating potential are associated with the first ten harmonics. This supports the possibility that the oscillation associated with 
Table 2. System parameters for Vela X-1 / GP Vel. The value for $K_{\mathrm{o}}$ is that resulting from fitting the phase bin means without a phase shift.

\begin{tabular}{|c|c|c|c|}
\hline Parameter & \multicolumn{2}{|c|}{ Value } & 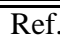 \\
\hline \multicolumn{4}{|l|}{ Observed } \\
\hline$a_{\mathrm{x}} \sin i /$ light sec & \multicolumn{2}{|c|}{$113.98 \pm 0.13$} & [1] \\
\hline$P / \mathrm{d}$ & \multicolumn{2}{|c|}{$8.964368 \pm 0.000040$} & [1] \\
\hline$T_{\pi / 2} / \mathrm{MJD}$ & \multicolumn{2}{|c|}{$48895.2186 \pm 0.0012$} & [1] \\
\hline$e^{\prime-x^{2}}$ & \multicolumn{2}{|c|}{$0.0898 \pm 0.0012$} & [1] \\
\hline$\omega / \operatorname{deg}$ & \multicolumn{2}{|c|}{$152.59 \pm 0.92$} & [1] \\
\hline$\theta_{\mathrm{e}} / \mathrm{deg}$ & \multicolumn{2}{|c|}{$33.8 \pm 1.3$} & [2] \\
\hline$\Omega$ & \multicolumn{2}{|c|}{$0.67 \pm 0.04$} & [3] \\
\hline$K_{\mathrm{o}} / \mathrm{km} \mathrm{s}^{-1}$ & \multicolumn{2}{|c|}{$22.6 \pm 1.5$} & [4] \\
\hline \multicolumn{4}{|l|}{ Inferred } \\
\hline$K_{\mathrm{x}} / \mathrm{km} \mathrm{s}^{-1}$ & \multicolumn{3}{|c|}{$278.1 \pm 0.3$} \\
\hline$T_{0} / \mathrm{MJD}$ & \multicolumn{3}{|c|}{$48896.777 \pm 0.009$} \\
\hline$q$ & \multicolumn{3}{|c|}{$0.081 \pm 0.005$} \\
\hline$\beta$ & 1.000 & $0.89 \pm 0.03$ & \\
\hline$i / \operatorname{deg}$ & $70.1 \pm 2.6$ & 90.0 & \\
\hline$M_{\mathrm{x}} / M_{\odot}$ & $2.27 \pm 0.17$ & $1.88 \pm 0.13$ & \\
\hline$M_{\mathrm{o}} / M_{\odot}$ & $27.9 \pm 1.3$ & $23.1 \pm 0.2$ & \\
\hline$a^{\prime}$ at periastron $/ R_{\odot}$ & $51.4 \pm 0.8$ & $48.3 \pm 0.3$ & \\
\hline$R_{\mathrm{L}}$ at periastron $/ R_{\odot}$ & $32.1 \pm 0.6$ & $30.2 \pm 0.2$ & \\
\hline$R_{\mathrm{o}} / R_{\odot}$ & $32.1 \pm 0.6$ & $26.8 \pm 0.9$ & \\
\hline
\end{tabular}

[1] Bildsten et al. (1997).

[2] Watson \& Griffiths (1977).

[3] Zuiderwijk (1995).

[4] This paper.

the frequencies $\approx f_{\text {orb }}$ and $\approx 4 f_{\text {orb }}$ may be induced by the tidal action of the neutron star.

An in-depth analysis of the possibility that a tidally excited oscillation mode exists in GP Vel requires an accurate knowledge of the stellar and orbital parameters. In particular, the internal structure of the B-type companion to the neutron star must be known to derive the spectrum of the eigenfrequencies present in the star. The radii quoted in Table 2 indicate that the B-type star might be in the Hertzsprung-gap. An additional complication arises from the unknown evolutionary history of the binary as the B-type star may be "polluted" by masstransfer episodes prior to the formation of the neutron star. In view of these complications, a detailed study of the oscillation spectrum of GP Vel and its possible use in an asteroseismological study is beyond the scope of our present investigation.

\section{Discussion}

Our final value for $K_{\mathrm{o}}$ of $22.6 \pm 1.5 \mathrm{~km} \mathrm{~s}^{-1}$ is slightly higher than, but comparable to, those quoted by other recent authors, noted in Sect. 2. Our result for the neutron star mass is compatible with van Kerkwijk et al.'s (1995) mass value of $M_{\mathrm{X}}=1.9_{-0.5}^{+0.7} M_{\odot}$, and that of Barziv et al. (2001), namely $M_{\mathrm{x}}=1.86 \pm 0.32 M_{\odot}$. We therefore support the view that the neutron star in Vela X-1 is more massive than the canonical value of $1.4 M_{\odot}$. However, there are a number of potential sources of systematic error, as noted in our earlier paper concerning the mass of the neutron star in Cen X-3 (Ash et al. 1999), some of which will be examined below.
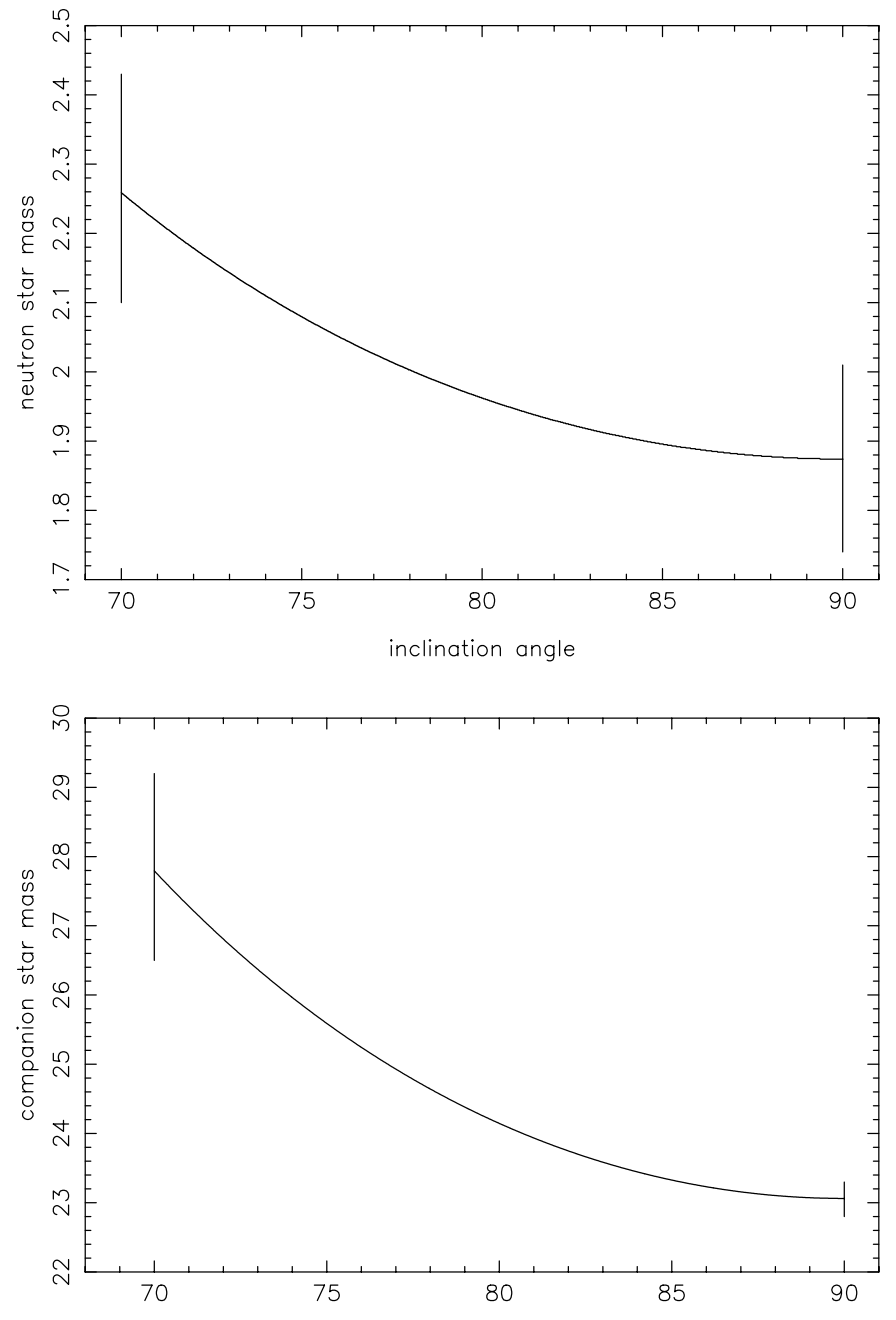

Fig. 8. The masses of the neutron star (upper panel) and companion star (lower panel) in solar units, as a function of inclination angle. The lower limit to the inclination angle is set by the condition that the companion star cannot over-fill its Roche lobe. Error bars on the masses at the extreme limits are determined as explained in the text.

\subsection{Stellar wind}

As GP Vel is an early-type star, it has a significant stellar wind, and a strong and variable stellar wind can have disastrous consequences when making radial velocity measurements, especially over an extended period of time. Barziv et al. (2001) made a detailed study of the effects of winds on their observations and showed that deviations in the Balmer lines in particular indicated the presence of a photo-ionization wake. We note that the supergiant GP Vel is of later type (B0) than that of Cen X-3 (O6-7), and thus has a weaker wind. Additionally, as our observations were made over a relatively short time period, the potential effect of long-term wind variation will be less than if the observations were spread over a number of years, as in the case of our Cen X-3 campaign, or over a year, as in the case of Barziv et al's Vela X-1 campaign. In other words, although the effect of the supergiant's stellar wind cannot be discounted, its effect should be less than its effect on our Cen X-3 results and less than on the results of Barziv et al. (2001). 
The apparent increase in amplitude of the radial velocity curve seen with increasing order in the Balmer series lines mentioned earlier, may also be an indication of a stellar wind. Conceivably, the different order lines have different contributions from the wind material as it moves further out, and this could produce the correlation seen. Since we have not used the Balmer lines in our analysis however, this effect does not influence our final result.

\subsection{X-ray heating}

The effect of X-ray heating of the surface of GP Vel may be compared with the corresponding effect in Cen X-3. The ratio of intrinsic to irradiated fluxes may be estimated for each system as $\left(L_{\mathrm{o}} / 4 \pi R_{\mathrm{o}}^{2}\right) /\left(L_{\mathrm{x}} / 4 \pi\left(a-R_{\mathrm{o}}\right)^{2}\right)$. The RXTE ASM lightcurves show that both sources have similar X-ray fluxes, but Cen X-3 is at over three times the distance of Vela X-1 (Sadakane et al. 1985; Humphreys \& Whelan 1975) so its $\mathrm{X}$-ray luminosity is at least a factor of ten higher. The luminosities of the supergiant stars in both systems are similar, but the radius of the star in Cen X-3 and the separation of the supergiant and neutron star are both around three times smaller in Cen X-3 (Ash et al. 1999) than in Vela X-1. Hence the effect of X-ray heating in Vela X-1 is of order ten times less than in Cen X-3. We were able to rule out significant $X$-ray heating in the case of Cen X-3 (Ash et al. 1999), and the effect should therefore be even less in this case.

\subsection{Deviations from Keplerian radial velocity curve}

Clearly, another source of uncertainty in the case of GP Vel arises from the observed deviations from a pure Keplerian radial velocity curve. van Kerkwijk et al. (1995) suggested that these deviations do not last longer than a single night, although our results (Fig. 3) suggest that they may last for up to two system orbits. They suggested that the deviations are the result of short-lived, high-order pulsations of the photosphere and we confirm that the frequencies appear to be harmonically related to the orbital frequency. It is possible therefore that they do represent tidally induced oscillations. As well as introducing another source of error into our attempts to measure the radial velocity of the centre of mass of the supergiant, the "wobbles" cause another problem. If the shape of the star is constantly changing, this will affect the extent to which the star fills its Roche lobe. Additionally, the size and shape of the Roche lobe itself will vary with phase, as the two components orbit each other in eccentric orbits. We believe that we have partially accounted for this effect by removing the $2.18 \mathrm{~d}$ modulation shown in Fig. 3.

However, as Fig. 7 demonstrates, there is clearly a remaining effect which may be characterised by an additional radial velocity variation at the orbital period. This manifests itself as a phase shift in the radial velocity curve with respect to the $\mathrm{X}$-ray orbital ephemeris. The measured amplitude of the radial velocity curve is therefore a combination of the orbital motion of GP Vel and an additional component that may be due to a non-radial pulsation induced by the eccentric orbit of the neutron star. There may even be another component of this non-radial pulsation at the orbital period that is in phase with the orbital motion and so undetectable. Our $K_{\mathrm{o}}$ value, and presumably all previous measurements too, are therefore subject to an unknown systematic error, and the stellar masses calculated from them are also uncertain because of this.

Another possibility for the origin of the extra velocity component at the orbital period is large-scale motion required to keep the tidal bulge pointing in the direction of the neutron star given the asynchronous stellar rotation $(\Omega=0.67)$. However, such a tidal bulge would extend in both directions - towards and away from the neutron star - and so the effect may cancel out.

As a further result, our identification of these departures from the radial velocity curve in GP Vel suggest that it may be the third recent candidate to exhibit tidally induced non-radial oscillations, after HD 177863 (De Cat 2001; Willems \& Aerts 2002), and HD 209295 (Handler et al. 2002).

\subsection{Neutron star mass}

The minimum neutron star mass consistent with our results is around $1.74 M_{\odot}$. A recent review of neutron star equations of state by Lattimer \& Prakash (2000) shows that such a mass would exclude the schematic potential models of Prakash, Ainsworth and Latimer, and the various models based on a field theoretical approach by Glendenning \& Moszkowski, by Glendenning and Schaffner-Bielich, and by Prakash et al. Hence the "softer" equations of state that incorporate components such as hyperons, kaons and quark matter are ruled out.

In order to be consistent with a neutron star mass of $\sim 1.4 M_{\odot}$, the radial velocity amplitude of GP Vel would have to be $K_{\mathrm{o}} \sim 18 \mathrm{~km} \mathrm{~s}^{-1}$, implying that the additional velocity component due to the non-radial pulsation has an amplitude of around $\sim 4.5 \mathrm{~km} \mathrm{~s}^{-1}$.

We conclude by noting that there are two large sources of uncertainty in deriving the masses of the stellar components. The first is that of the value $\beta$ (the ratio of the radius of the companion star to its Roche lobe radius). This in turn leads to a large uncertainty in $\sin i$ (and hence the masses) and is unlikely to be reduced even with optical spectroscopic observations of greater signal-to-noise. The second source of uncertainty is the additional radial velocity component at the orbital period that we have discovered and represented by a phase shift. Removing the effect of such a velocity component is likely to require detailed modelling of tidally excited oscillation modes in GP Vel which in turn requires an accurate knowledge of the stellar parameters. Consequently, the mass measurement presented here may represent the best that is achievable.

Acknowledgements. We thank the Mount Stromlo Observatory staff for maintaining the 74-inch telescope. The data analysis reported here was carried out using facilities provided by PPARC, Starlink and the OU research committee. HQ was employed under PPARC grant number GR/L64621 during the course of this work. The authors would also like to thank Deepto Chakrabarty for his help, and the referee, Marten van Kerkwijk, for his many detailed and constructive suggestions which have helped to significantly improve the paper. 


\section{Appendix}

Table A.1. The radial velocities of GP Vel relative to that of $\epsilon$ Ori.

\begin{tabular}{|c|c|c|c|c|c|}
\hline HJD - 2450000 & $R V / \mathrm{km} \mathrm{s}^{-1}$ & HJD - 2450000 & $R V / \mathrm{km} \mathrm{s}^{-1}$ & HJD - 2450000 & $R V / \mathrm{km} \mathrm{s}^{-1}$ \\
\hline 115.9492 & $-39.978 \pm 2.229$ & 123.9840 & $-52.446 \pm 3.876$ & 129.1373 & $-68.519 \pm 2.676$ \\
\hline 115.9620 & $-39.016 \pm 2.237$ & 124.0222 & $-52.899 \pm 1.868$ & 129.1820 & $-69.463 \pm 2.743$ \\
\hline 115.9900 & $-39.473 \pm 2.130$ & 124.0368 & $-54.319 \pm 2.242$ & 129.1951 & $-68.887 \pm 2.855$ \\
\hline 116.0028 & $-38.093 \pm 2.047$ & 124.0507 & $-54.361 \pm 2.167$ & 129.2080 & $-69.592 \pm 4.292$ \\
\hline 116.0153 & $-38.126 \pm 2.148$ & 124.1014 & $-52.954 \pm 2.206$ & 129.2211 & $-69.514 \pm 3.050$ \\
\hline 116.1147 & $-35.260 \pm 2.002$ & 124.1153 & $-52.398 \pm 1.780$ & 129.2344 & $-67.797 \pm 3.757$ \\
\hline 116.1273 & $-34.446 \pm 1.959$ & 124.1292 & $-51.638 \pm 2.230$ & 129.9134 & $-70.298 \pm 2.184$ \\
\hline 116.1400 & $-33.855 \pm 1.990$ & 124.1798 & $-52.602 \pm 2.338$ & 129.9264 & $-70.958 \pm 2.530$ \\
\hline 116.1643 & $-33.359 \pm 2.026$ & 124.1944 & $-50.835 \pm 2.627$ & 129.9393 & $-70.168 \pm 2.452$ \\
\hline 116.1768 & $-32.720 \pm 2.218$ & 124.2083 & $-51.151 \pm 3.466$ & 129.9995 & $-70.815 \pm 2.380$ \\
\hline 116.1905 & $-32.454 \pm 2.090$ & 124.9819 & $-30.123 \pm 2.138$ & 130.0124 & $-71.690 \pm 2.064$ \\
\hline 116.9187 & $-31.398 \pm 2.249$ & 124.9944 & $-29.702 \pm 2.094$ & 130.0251 & $-70.973 \pm 2.494$ \\
\hline 116.9314 & $-30.109 \pm 2.933$ & 125.0078 & $-29.527 \pm 2.370$ & 130.0515 & $-71.616 \pm 2.018$ \\
\hline 116.9468 & $-30.908 \pm 3.410$ & 125.1431 & $-27.910 \pm 2.830$ & 130.0650 & $-71.685 \pm 2.830$ \\
\hline 117.0059 & $-34.455 \pm 5.798$ & 125.1561 & $-28.457 \pm 3.213$ & 130.0780 & $-72.801 \pm 3.691$ \\
\hline 117.9872 & $-46.720 \pm 3.585$ & 125.1691 & $-26.982 \pm 2.189$ & 130.1179 & $-70.146 \pm 2.440$ \\
\hline 118.0148 & $-47.951 \pm 6.251$ & 125.9233 & $-34.387 \pm 2.657$ & 130.1314 & $-69.869 \pm 2.277$ \\
\hline 118.0710 & $-48.042 \pm 3.267$ & 125.9373 & $-34.510 \pm 2.742$ & 130.1447 & $-69.998 \pm 2.608$ \\
\hline 118.0877 & $-51.834 \pm 5.119$ & 125.9506 & $-34.692 \pm 2.958$ & 130.1865 & $-69.660 \pm 3.002$ \\
\hline 118.1010 & $-53.321 \pm 7.217$ & 126.0339 & $-33.610 \pm 2.408$ & 130.1996 & $-70.063 \pm 2.521$ \\
\hline 118.1454 & $-50.003 \pm 2.466$ & 126.0469 & $-33.979 \pm 2.359$ & 130.2126 & $-70.230 \pm 3.305$ \\
\hline 118.1585 & $-48.447 \pm 5.900$ & 126.0611 & $-33.347 \pm 2.395$ & 131.9124 & $-61.202 \pm 2.797$ \\
\hline 118.1775 & $-53.136 \pm 3.112$ & 126.0743 & $-33.101 \pm 2.326$ & 131.9254 & $-62.224 \pm 3.068$ \\
\hline 118.9354 & $-49.826 \pm 3.501$ & 126.1222 & $-32.552 \pm 2.343$ & 131.9384 & $-58.133 \pm 2.653$ \\
\hline 118.9478 & $-49.141 \pm 3.659$ & 126.1352 & $-31.563 \pm 2.088$ & 131.9514 & $-59.732 \pm 3.326$ \\
\hline 118.9889 & $-50.111 \pm 3.441$ & 126.1482 & $-31.939 \pm 2.511$ & 131.9805 & $-57.723 \pm 3.156$ \\
\hline 119.0014 & $-50.441 \pm 3.921$ & 126.1848 & $-31.483 \pm 2.923$ & 132.0066 & $-57.949 \pm 3.367$ \\
\hline 119.0138 & $-52.065 \pm 3.781$ & 126.1977 & $-30.767 \pm 2.565$ & 132.0322 & $-56.251 \pm 2.923$ \\
\hline 119.0552 & $-51.831 \pm 3.906$ & 126.2107 & $-31.893 \pm 2.842$ & 132.0453 & $-54.676 \pm 2.862$ \\
\hline 119.0677 & $-53.398 \pm 3.627$ & 126.9419 & $-36.272 \pm 2.603$ & 132.0583 & $-57.021 \pm 2.694$ \\
\hline 119.0802 & $-54.218 \pm 4.755$ & 126.9683 & $-35.469 \pm 2.878$ & 132.0735 & $-55.879 \pm 2.755$ \\
\hline 119.1138 & $-55.934 \pm 3.703$ & 127.0924 & $-39.613 \pm 2.182$ & 132.0864 & $-55.474 \pm 2.963$ \\
\hline 119.1269 & $-54.718 \pm 3.383$ & 127.1062 & $-39.544 \pm 3.559$ & 132.0995 & $-52.365 \pm 3.715$ \\
\hline 119.1394 & $-54.888 \pm 3.520$ & 127.1201 & $-38.081 \pm 2.531$ & 132.1382 & $-53.565 \pm 3.106$ \\
\hline 119.9193 & $-84.329 \pm 8.318$ & 127.1465 & $-40.720 \pm 2.797$ & 132.1517 & $-53.728 \pm 3.029$ \\
\hline 119.9325 & $-75.408 \pm 3.151$ & 127.1601 & $-38.836 \pm 2.407$ & 132.1713 & $-53.901 \pm 3.810$ \\
\hline 120.0306 & $-76.237 \pm 2.404$ & 127.1734 & $-41.524 \pm 2.751$ & 132.2137 & $-49.692 \pm 3.727$ \\
\hline 120.0431 & $-75.741 \pm 2.576$ & 127.2103 & $-41.617 \pm 3.177$ & 132.2267 & $-54.489 \pm 3.764$ \\
\hline 120.0557 & $-75.138 \pm 2.346$ & 127.2234 & $-41.710 \pm 3.056$ & 132.2397 & $-51.464 \pm 3.900$ \\
\hline 120.1009 & $-75.197 \pm 2.393$ & 127.9268 & $-58.126 \pm 4.144$ & 132.9132 & $-59.635 \pm 3.026$ \\
\hline 120.1135 & $-74.375 \pm 2.652$ & 127.9398 & $-59.568 \pm 3.727$ & 132.9274 & $-57.425 \pm 3.343$ \\
\hline 120.1260 & $-75.235 \pm 2.312$ & 127.9528 & $-59.855 \pm 4.283$ & 132.9404 & $-56.218 \pm 2.664$ \\
\hline 120.1824 & $-75.016 \pm 2.495$ & 128.0551 & $-62.303 \pm 4.473$ & 133.0305 & $-54.996 \pm 2.741$ \\
\hline 120.1962 & $-74.390 \pm 2.519$ & 128.0698 & $-61.858 \pm 4.231$ & 133.0435 & $-53.878 \pm 2.518$ \\
\hline 120.2103 & $-74.013 \pm 2.769$ & 128.0829 & $-61.410 \pm 4.222$ & 133.0570 & $-54.587 \pm 2.539$ \\
\hline 120.2417 & $-72.921 \pm 2.729$ & 128.1450 & $-64.184 \pm 4.351$ & 133.9029 & $-35.155 \pm 3.759$ \\
\hline 120.2543 & $-72.066 \pm 2.847$ & 128.1585 & $-61.980 \pm 3.912$ & 133.9158 & $-36.872 \pm 3.280$ \\
\hline 120.2669 & $-74.260 \pm 3.256$ & 128.1715 & $-60.965 \pm 4.288$ & 133.9288 & $-37.601 \pm 3.099$ \\
\hline 120.9177 & $-69.011 \pm 2.892$ & 128.1846 & $-65.137 \pm 3.990$ & 133.9773 & $-36.505 \pm 3.376$ \\
\hline 120.9298 & $-67.862 \pm 2.769$ & 128.2014 & $-63.062 \pm 3.888$ & 133.9903 & $-35.226 \pm 3.121$ \\
\hline 120.9430 & $-68.414 \pm 2.985$ & 128.2145 & $-63.871 \pm 4.560$ & 134.0064 & $-36.135 \pm 3.172$ \\
\hline 120.9659 & $-67.933 \pm 2.808$ & 128.2275 & $-63.709 \pm 4.447$ & 134.0861 & $-37.570 \pm 3.744$ \\
\hline 120.9789 & $-68.716 \pm 2.809$ & 128.9267 & $-69.557 \pm 3.368$ & 134.9085 & $-28.154 \pm 3.774$ \\
\hline 120.9929 & $-67.612 \pm 2.848$ & 128.9396 & $-69.768 \pm 3.061$ & 134.9215 & $-29.949 \pm 2.582$ \\
\hline 121.0343 & $-67.387 \pm 2.906$ & 128.9526 & $-70.041 \pm 3.134$ & 134.9346 & $-29.342 \pm 2.227$ \\
\hline 122.1584 & $-68.859 \pm 5.309$ & 129.0007 & $-68.518 \pm 3.256$ & 134.9852 & $-28.680 \pm 2.028$ \\
\hline 122.1803 & $-76.832 \pm 5.303$ & 129.0142 & $-70.118 \pm 3.534$ & 134.9982 & $-29.925 \pm 2.816$ \\
\hline 122.1934 & $-70.372 \pm 3.793$ & 129.0272 & $-68.765 \pm 3.262$ & 135.0146 & $-29.764 \pm 2.670$ \\
\hline 123.9486 & $-55.418 \pm 2.464$ & 129.1112 & $-68.900 \pm 2.794$ & 135.0596 & $-30.751 \pm 3.978$ \\
\hline 123.9680 & $-53.818 \pm 2.188$ & 129.1244 & $-68.574 \pm 2.835$ & & \\
\hline
\end{tabular}




\section{References}

Ash, T. D. C., Reynolds, A. P., Roche, P., et al. 1999, MNRAS, 307, 357

Barziv, O., Kaper, L., van Kerkwijk, M. H., Telting, J. H., \& van Paradijs, J. 2001 A\&A, 377, 925

Bildsten, L., Chakrabarty, D., Chiu, J., et al. 1997, ApJS, 113, 367

Brucato, R. J., \& Kristian, J. 1972, ApJ, 173, L105

De Cat, P. 2001, An observational study of bright southern slowly pulsating B stars, Ph.D. Thesis, Katholieke Universiteit Leuven, Belgium

Chakrabarty, D., Wang, Z., Juett, A., Lee, J., \& Roche, P. 2002, ApJ, 573,789

Charles, P. A., Mason, K. O., Culhane, J. L., Sanford, P. W., \& White, N. E. 1976, X-ray Binaries, NASA SP-389, 629

Chodil, G., Mark, H., Rodrigues, R., Seward, F. D., \& Swift, C. D. 1967, ApJ, 150, 57

Clark, J. S., Goodwin, S. P., Crowther, P. A., et al. 2002, A\&A, 392, 909

Deeter, J. E., Boynton, P. E., Shibazaki, N., et al. 1987, AJ, 93, 877

Forman, W., Jones, C., Tananbaum, H., et al. 1973, ApJ, 182, L103

Giacconi, R., Murray, S., Gursky, H., et al. 1972, ApJ, 178, 281

Handler, G., Balona, L. A., Shobbrook, R. R., et al. 2002, MNRAS, 333,262

Hiltner, W. A., Werner, J., \& Osmer, P. 1972, ApJ, 175, L19

Humphreys, R. M., \& Whelan, J. 1975, Observatory, 95, 171

Lattimer, J. M., \& Prakash, M. 2000, Phys. Rep., 333-334, 121
McClintock, J. E., Rappaport, S., Joss, P. C., et al. 1976, ApJ, 206, L99

Polfliet, R., \& Smeyers, P. 1990, A\&A, 237, 110

Rappaport, S. A., \& Joss, P. C. 1983, in Accretion Driven Stellar X-ray Sources, ed. W. H. G. Lewin, \& E. P. J. van den Heuvel (Cambridge University Press)

Rappaport, S. A., \& McClintock, J. 1975, IAUC 2833

Rappaport, S. A., Joss, P. C., \& McClintock, J. E. 1976, ApJ, 206, L103

Rappaport, S. A., Joss, P. C., \& Stothers, R. 1980, ApJ, 235, 570

Sadakane, K., Hirata, R., Jugaku, J., et al. 1985, ApJ, 288, 284

Stickland, D., Lloyd, C., \& Radziun-Woodham, A. 1997, MNRAS, 286, L21-24

Ulmer, M. P., Baity, W. A., Wheaton, W. A., \& Peterson, L. E. 1972, ApJ, 178, L121

van Kerkwijk, M. H., van Paradijs, J., \& Zuiderwijk, E. J., et al. 1995, A\&A, 303, 483

van Paradijs, J. A., Hammerschlag-Hensberge, G., van den Heuvel, E. P. J., et al. 1976, Nature, 259, 547

van Paradijs, J. A., Zuiderwijk, E. J., Takens, R. J., \& Hammerschlag-Hensberge, G. 1977, A\&AS, 30, 195

Wallerstein, G. 1974, ApJ, 194, 451

Watson, M. G., \& Griffiths, R. E. 1977, MNRAS, 178, 513

Willems, B., \& Aerts, C. 2002, A\&A, 384, 441

Wilson, R. E., \& Terrell, D. 1998, MNRAS, 296, 33

Zuiderwijk, E. J. 1995, A\&A, 299, 79

Zuiderwijk, E. J., van den Heuvel, E. P. J., \& Hensberge, G. 1974, A\&A, 35, 353 